\title{
Tele-Mental Health Utilization Among People with Mental Illness to Access Care During the COVID-19 Pandemic
}

\author{
Mark Costa ${ }^{1}\left[\right.$ Graziela Reis $^{1} \cdot$ Anthony Pavlo $^{1} \cdot$ Chyrell Bellamy $^{1} \cdot$ Katherine Ponte $^{1,2} \cdot$ Larry Davidson $^{1}$
}

Received: 12 August 2020 / Accepted: 29 January 2021 / Published online: 10 February 2021

(C) The Author(s), under exclusive licence to Springer Science+Business Media, LLC part of Springer Nature 2021

\begin{abstract}
Shortly after the COVID-19 pandemic arrived at the United States, mental health services moved towards using tele-mental health to provide care. A survey about resilience and tele-mental health was developed and conducted with ForLikeMinds' members and followers. Correlational analysis was used to examine relationships between quantitative variables. A phenomenological approach was used to analyze open questions responses. Sixteen percent of participants were coping well with the pandemic; 50\% were coping okay; and 34\% said that they were coping poorly. Three main themes emerged from the qualitative analysis: accessibility to care; self-care strategies; and community support and relationship. The responses from participants seems to reflect the combination of two main factors-the challenges they were facing in accessing care through tele-mental health plus the mental health consequences from COVID-19. This survey reflects the importance of building innovative strategies to create a working alliance with people who need care through tele-mental health.
\end{abstract}

Keywords Mental illness $\cdot$ COVID-19 $\cdot$ Tele-mental health $\cdot$ Resilience $\cdot$ Social isolation

\section{Introduction}

In the United States, between March 10, 2020, and March 27, 2020, all states implemented some form of social distancing and/or shelter in place policy in response to the COVID-19 pandemic (Siedner et al. 2020). Mental health care outpatient clinics and services rapidly moved towards using tele-mental health to comply with social distancing policies and to protect their clients and staff from COVID-19 (Wright and Caudill 2020). This fast move was supported by the fact that mental health care via tele-mental health (also referred to as telepsychiatry) has been proven to be effective (Shore 2013; Chakrabarti 2015; Torous et al. 2020). To facilitate this move, emergency waivers suspending the requirement to comply with the Health Insurance Portability and Accountability Act of 1996 (HIPAA - the health information privacy law in the US) for tele-mental health were issued by governmental agencies (Wright and Caudill

Mark Costa

mark.costa@yale.edu

1 Department of Psychiatry, Yale University School of Medicine, Program for Recovery and Community Health. New Haven, New Haven, CT, USA

2 ForLikeMinds, New York, NY, USA
2020). In this process, the fidelity that helped establish the effectiveness of telehealth/telemedicine might have been lost. For instance, the guide for tele-mental health developed by the American Psychiatric Association together with the American Telemedicine Association states that "all modes of communication of personal health history shall be HIPAA compliant", as well as the need to ensure privacy (Shore et al. 2018). With the emergency HIPPA waivers, these recommendations could, potentially, not be followed with the same rigor, leading to decrease in quality of service. Even before the current COVID-19 pandemic, several challenges and concerns have been raised regarding tele-mental health as a substitute to in-person appointments, (e.g. anxiety and technophobia) (Langarizadeh et al. 2017). This strategy, at most, should be considered an excellent adjunct to traditional service delivery, complementing and supplementing in-person care (Chakrabarti 2015; Mehrotra et al. 2017; Greenhalgh et al. 2018). After two months of social distancing policies where telehealth/telemedicine became the new norm for outpatient mental health service delivery, ForLikeMinds, conducted an on-line survey with its community about tele-mental health. ForLikeMinds is an online peer support community dedicated to the recovery and wellness of people living with or supporting someone with mental illness, substance use, or stressful life events (Ponte 2020). 
The utilization of telecommunication platforms, such as videoconferencing, to deliver mental health services remotely defines tele-mental health (Mahmoud et al. 2020). It was not a surprise, then, that mental health services via tele-mental health became a routine component during the COVID19 pandemic. Tele-mental health is a consensus choice when it comes to providing a safe and convenient access to routine mental healthcare, avoiding exposure to COVID-19, especially for those at higher risk of being affected (Smith et al. 2020). In recent years, there has been a significant expansion of tele-mental health services. For instance, in the US in 2017 almost $30 \%$ of mental health facilities offered tele-mental health services (Mahmoud et al. 2020).

Tele-mental health is a well-known practice and has been around for more than half a century (Mahmoud et al. 2020; Hilty et al. 2013). In 2009, the American Telemedicine Association established guidelines for tele-mental health. In their guidelines, standard provisions include the availability of appropriate staff before, during, and after tele-mental health encounters to meet patient and provider needs and enhanced requirements for privacy and confidentiality (Yellowlees et al. 2010). In their review of the literature about the effectiveness of tele-mental health, Hilty et al. (2013) concluded that tele-mental health services were mostly effective if a plan of assessment and care for patients was in place. Other key administrative issues for successful tele-mental health program implementation would be licensure requirements, malpractice insurance, insurance coverage, adherence to HIPPA regulations, and establishment of protocols (Write and Caudill 2020). On the other hand, several limitations to tele-mental health have been identified such as lower quality of patient-physician relationship, fragmentation of care, and challenges in accessing telecommunication technology (Dorsey and Topol 2016; Greenhalgh et al. 2020).

A review of the literature before the COVID-19 pandemic demonstrated that, regarding psychotherapeutic interventions and therapeutic alliance in treatment, it seems that patient satisfaction with tele-mental health was comparable to in-person delivery, when well implemented and technology support was available (Jenkins-Guarnieri et al. 2015).

\section{Research Question}

In their first on-line survey about the COVID-19 pandemic and the concerns of people with mental illness, ForLikeMinds captured significant concerns about how the pandemic was affecting respondents' mental health. Participants in the first survey were specifically concerned with disruption in treatment, access to medication, and the likelihood of their condition getting worse and/or developing a new mental illness (Costa et al. 2020). Leadership at ForLikeMinds decided to conduct a second on-line survey to address resilience and mental health care during the COVID-19 pandemic. After almost two months since the beginning of social isolation measures, how were people with mental illness doing? Was tele-mental health working for people with mental illness? Did they have any suggestions that could improve tele-mental health?

\section{Methodology}

A survey about resilience and tele-mental health-the Coronavirus Mental Health Resilience Survey-was developed by ForLikeMinds' leadership based on their own research and perception of how their network was coping with the pandemic. It was then sent out to their members and followers. The Coronavirus Mental Health Resilience Survey is composed of 11 multiple-choice questions with the option of comments and one open question. The multiple-choice questions addressed the following issues: did participants self-identify as living with a mental illness, which mental illness they identified with, where they were in their recovery journey, what was the impact of the pandemic on their mental health, if they had developed a new mental illness, how had the mental health support they received changed during the pandemic, how had their mental health care changed as a consequence of the pandemic, if they were receiving tele-mental health support, how they were coping with the pandemic, how isolated they felt, and how had their level of social connectedness changed during the pandemic. The open-ended question asked participants about their thoughts on what would be most helpful for their communities during this difficult time.

The survey was distributed to members of ForLikeMinds and to their Facebook community using Survey Monkey. The dataset extracted from Survey Monkey was de-identified before it was sent to the Yale Program for Recovery and Community Health for analysis (i.e., secondary data analysis). SPSS was used for the statistical analysis. Analyses were run after cases were selected. Case selection was based on having checked the statement "I am completing this survey primarily as someone living with mental illness". Frequencies were determined for each multiple-choice question. Bivariate Correlations utilizing Spearman rank order correlation was established to determine the correlations between the variable "how do you feel you are coping with the pandemic" (ordinal variable) and all other nominal and ordinal variables. Significance (2-tailed) was established at a level of $p<0.05$. Effect sizes of the relationship between variables were established and an effect size of approximately 0.1 was considered a small effect size, and effect size of approximately 0.3 was considered a medium effect size and 
effect size of approximately 0.5 was considered a large effect size Swank and Mullen (2017).

Comments to the question "have you seen your mental healthcare provider by video session" were analyzed together with the responses to the open-ended question "what would help our community most during this crisis?". A phenomenological approach was used to analyze each individual statement and to define a main theme for each statement (Davidson 2003; Davidson et al. 2008; Van Manen 2016). Statements were also classified as positive, neutral, or negative. Two members of the research group analyzed and defined themes for each statement independently. Themes generated from statements were then grouped based on how participants felt they were coping with the pandemic. Classification of positive, neutral, and negative, was based on the interpretation of the researchers who analyzed the statements. Both researchers agreed in how each statement were classified. Results from the independent analysis were compared and a final set of themes were established.

Institutional Review Board (IRB) was not pursued because there was no identifiable private health information in the data set that researchers used for the analysis.

\section{Results}

ForLikeMinds is an online support community dedicated to the recovery and wellness of people living with or supporting someone with mental illness, substance use, or stressful life events. It has over 15,000 members plus a Facebook community of nearly 23,000 followers. Four hundred and thirty-five unique individuals responded to the survey. Of these, 381 individuals (88\%) self-identified as living with a mental illness. As mentioned in the methodology, all data analysis reported here refers to participants living with a mental illness. Of those living with a mental illness, 74\% reported having an anxiety disorder, $60 \%$ having a major depressive disorder, $36 \%$ having post-traumatic stress disorder, $30 \%$ having bipolar disorder, $13 \%$ having borderline disorder, $11 \%$ having obsessive compulsive disorder, and $5 \%$ having schizophrenia (respondents could report multiple conditions). Seventy one percent (71\%) of participants said they had at least two mental illnesses and 59\% said they had at least three mental illnesses. The most likely conditions to occur together were anxiety and major depressive disorder (49\% of participants had both conditions), anxiety and post-traumatic stress disorder (31\%), and major depressive disorder and post-traumatic depressive disorder $(26 \%)$. When asked about where they were in their recovery, $17 \%$ responded that they were living a full and meaningful life, $36 \%$ said that they had symptoms but were able to manage them, and $47 \%$ said that they were struggling to get or stay well.
The Coronavirus Mental Health Resilience Survey didn't ask specific sociodemographic questions. Based on other surveys conducted at ForLikeMinds which collected sociodemographic data, we expect respondents to be mostly females $(85 \%)$, white $(84 \%)$ and 55 years old and older (57\%).

Participants were asked about how much the COVID-19 pandemic had impacted their mental health. For $21 \%$, their mental health was much worse, $51 \%$ said their mental health was worse, $22 \%$ said that the pandemic had no real impact on their mental health, $5 \%$ said that they were doing better, and $1 \%$ said that their mental health had been much better since the pandemic. When asked if they had developed a "new" mental illness during the COVID-19 pandemic, 21\% responded affirmatively. Regarding the mental health supports they received, $12 \%$ said that their mental health support stopped after the pandemic started, $30 \%$ said that it had decreased, $42 \%$ said their mental health support had remained unchanged and for $16 \%$ their mental health support had actually increased. Changes in mental health care was addressed in the survey. For 7\%, their mental health care improved during the pandemic, $55 \%$ said that their care remained unchanged, while $28 \%$ said that their care deteriorated once the pandemic had started. Additionally, $7 \%$ said that it had become more difficult for them to get their medication.

For the question about seeing their mental health care provider through video conferencing following the stay at home recommendations, participants were given the option of checking more than one response. With this, $8 \%$ said that they started seeing a new mental health care provider by video sessions, 33\% said that they had switched from inperson sessions to video sessions with the same provider, $33 \%$ said that they preferred in-person sessions to video sessions, $3 \%$ said that they preferred video sessions to in-person sessions, about $8 \%$ said that they would like to continue seeing their mental health care provider by video sessions after the stay at home recommendations are lifted, about $10 \%$ of participants reported that they were meeting with their providers over the phone and $11 \%$ said that they had stopped seeing their mental health care provider because tele-mental health was not offered to them.

Participants were asked about how they were coping with the pandemic. For $16 \%$, they were coping well; $50 \%$ said that they were coping okay; and 34\% said that they were coping poorly. When asked about how isolated they felt compared to before the pandemic, $69 \%$ said that they were feeling more isolated, $26 \%$ felt the same, and $4 \%$ said that they felt less isolated. In response to the question about how the level of social connectedness had changed for them during the pandemic, $64 \%$ said that they felt less connected socially, $25 \%$ felt the same, and $11 \%$ felt that their level of social connectedness had increased. 
Several variables presented a significant correlation with how people felt they were coping with the pandemic. Of notice, the variables that captured mental health treatment and how people felt about their mental health treatment during the pandemic presented a moderate to strong correlation with how people felt they were coping with the pandemic. For instance, those who saw the same provider through video sessions were coping better with the pandemic, while those who felt that their mental health treatment had deteriorated or who had more difficulty in getting their medication or who felt that their mental health support had stopped were coping worse. Also of notice, those who said that they had anxiety disorder or major depressive disorder or PTSD were coping worse while those who said that they had bipolar disorder were coping better (Table 1).

One hundred and seventy-five participants (46\%) of those living with a mental illness provided a written response to the two analyzed open questions. Altogether, 205 written statements were analyzed. Eighty-nine participants (23\%) provided additional comments for the question "have you seen your mental health care provider by videos session during the lockdown" (question 1) and 116 participants (30\%) answered the question "what would help our community most during this crisis?" (question 2).

Regarding the classification of each statement, 52 statements $(30 \%)$ were classified as negative (e.g. "I am not sleeping well. I am often sad. I find it difficult to get things done."), 69 (39\%) were classified as neutral (e.g. "Depends on the day"), and 84 (48\%) were classified as positive (e.g. "I have never felt more rested and in control than I do right now"). Convergence was observed between multiple choice and open-ended questions and qualitative analysis was conducted taking convergence into consideration.

Three main themes emerged from the analysis: (1) accessibility to care; (2) self-care strategies; and (3) community support and relationship.

Related to accessibility to care, those who said that they were coping poorly with the pandemic presented several barriers and challenges such as not feeling comfortable using tele-mental health, not having been able to connect to their provider, feeling that there was a decrease in number of sessions, challenges in using technology, and that the phone was less effective:

"I can't do video sessions. I don't have internet, and data doesn't work. This is hard [because] as a kid I was sexually abused (...) and I just can't do that. Every time I think about it I want to puke and it's led to flashbacks about the videos and abuse, and I just can't do it..."

"I am hearing impaired. Video Conferencing is difficult."

"Instead of 50 minutes of therapy it's 30-minute phone sessions, no video."

They also presented suggestions such as group therapy via video and more options for online counseling:

"More options for online counseling. Most counselors are not familiar with or comfortable using technology such as FaceTime or Zoom. Also, insurance should cover these sessions just as it does in-person sessions."
Table 1 Variables that significantly correlated with how people felt they were coping with the pandemic

\begin{tabular}{lrr}
\hline Variables & Correlation coefficient & $p$ value \\
\hline Positive correlations & & \\
Bipolar disorder & 0.16 & 0.003 \\
Where are you on your recovery & 0.47 & $<0.001$ \\
Mental health treatment has improved & 0.17 & $<0.001$ \\
Mental health treatment was unchanged & 0.25 & $<0.001$ \\
Seeing same provider through video sessions & 0.37 & $<0.001$ \\
Mental health support was unchanged or increased & 0.24 & $<0.001$ \\
Feeling better or much better after the pandemic & 0.61 & $<0.001$ \\
Feeling more connected socially after the pandemic & 0.16 & 0.003 \\
Negative correlations & & $<0.001$ \\
Anxiety disorder & -0.26 & 0.002 \\
Major depressive disorder & -0.16 & 0.006 \\
PTSD & -0.14 & $<0.001$ \\
Having developed a new mental illness & -0.27 & $<0.001$ \\
Mental health treatment has deteriorated & -0.29 & 0.01 \\
It has been more difficult to get medication & -0.14 & 0.003 \\
Mental health support has stopped & -0.16 & $<0.001$ \\
Feeling more isolated & -0.37 &
\end{tabular}


For those coping satisfactorily with the pandemic, accessibility to care fell into two subcategories: some respondents were meeting with their providers over the phone instead of on video sessions, but that did not pose much of a problem; others were still waiting for their first tele-mental health session:

"Don't have access to video sessions. We have phone sessions and would like the option to choose whether we'd like phone sessions or in person sessions after the restrictions are lifted."

"Telephone appointments are the norm in our area."

"Waiting for the sessions with new mental health care provider."

For those who were coping well with the pandemic many felt very supported by their providers, but they also pointed out limits of tele-mental health:

"I have had phone consults. My doctor calls me anytime I leave a message. Before and during this pandemic."

"Some conditions are less likely to deal with screen time and can actually go downhill as a result: concussions or with dementia can get more confused. The technology has not gotten easier, and with older folks particularly affected the tech has not helped."

For the two other main themes, self-care strategies and community support and relationships, comments and suggestions were similar regardless of how participants said they were coping with the pandemic. Participants suggested self-care strategies that included having hope, faith, mindfulness, and education for better self-care:

"More freedom and personal accountability ... reminders it is each individual's responsibility to stay clean and healthy."- participant who was coping poorly "I believe to try to make videos easy to understand of mindfulness or other skills to try calm ourselves down and also try to connect and reach out to each person by their culture and/or lifestyle so each person can try to be informed about mental illness/health and so they can better reach to use mental health services." - participant who was coping okay

"Education on resources for people experiencing more anxiety or other mental health issues." - participant who was coping well

Regarding community support and relationships, concerns with loneliness and lack of support was pervasive throughout all three groups:

"Recognition that isolation disproportionately affects some people, including those who were already struggling with loneliness; it is distinct from the stress that everyone is facing and has tangible effects." (coping poorly participant)

"We need to connect physically with real people. Online is not a substitute. Superficial conversations at a distance is not a substitute." (coping okay)

"Seeing people, touching people through hugs. I never get hugs. Ever. More realistically, if someone could just stop by without coming in, just to say hi." (coping well)

They also suggested different community support strategies directed towards increase in relationships and connection to others such as doing Zoom activities together, a place to connect with others, and webinars about how to reach out and have social contact via internet.

Results from the quantitative and qualitative responses seems to agree or converge.

\section{Discussion}

The severity and unprecedented nature of the COVID-19 pandemic imposed the need for bold and creative solutions to address the disruption of access to mental health care, principally after social distancing and stay at home policies were abruptly implemented. Tele-mental health became a very important form of delivering mental health care in this new scenario. On the one hand, this survey shows that the tele-mental health received by participants, in many cases, had not adhered to the fidelity criteria of the evidence-based model for tele-mental health. This seems to be especially true regarding recommendations related to availability of staff before, during, and after the tele-mental health appointment (Yellowlees et al. 2010). People who do not have access to technology, who do not have the knowledge, who are afraid for different reasons of using tele-mental health, or who do not have enough privacy in their homes were left with few options (Sevelius et al. 2020). A significant group of participants are talking to their providers only over the phone (at least $10 \%$ ) and at least $11 \%$ said that they had stopped seeing their mental health care provider. When responding to preferences, $33 \%$ said that they preferred in-person sessions to video sessions, while only $3 \%$ said that they preferred video sessions. On the other hand, for many participants, the COVID-19 pandemic seems to have aggravated their lives. Almost $75 \%$ said they had anxiety, $72 \%$ said that their mental health was worse or much worse since the pandemic, and $21 \%$ said that they had developed a new mental illness during the pandemic. The response from participants about how they were coping with the pandemic seems to reflect the combination of these two main factors - the challenges they were facing in accessing care 
through tele-mental health plus the mental health consequences from COVID-19. Not surprising, the effect size of the correlation between how participants were coping with the pandemic and feeling more isolated was medium to large and the effect size between coping and where they were in their recovery journey was large Swank and Mullen (2017). For participants, the tele-mental health they received could be improved by offering more options of care and by increasing the quality of care provided. Making it easier to connect to providers, whether by offering technical assistance or by decreasing the waiting time for an appointment was also key. Finally, participants suggested that tele-mental health could be a very useful way to help people learn self-care strategies (e.g. mindfulness) and to connect with other people to help build relationships and to address loneliness.

People described feeling disconnected from others, including both family and friends and mental health professionals, and may have felt abandoned by the mental health system and government. People described less personal contact with others. Those interacting with friends, family, and mental health professionals via phone or video said these connections were not as good, or were not of the same quality, as in-person interactions. Respondents also described not feeling cared for by the mental health system. For instance, tele-mental health could be more utilized for self-care strategies and to help build community supports and relationships. These could be fruitful ways of further developing and refining individual preferences in utilizing tele-mental health (Horowitz et al. 2006).

\section{Study Limitation}

Respondents to the survey represent only a small percentage of ForLikeMinds community. It was not possible to determine if this sample is an unbiased representation of the whole ForLikeMinds community. There might be a selection bias in the sampling methodology. As we used social media to recruit and for people to respond to the survey, we could have a biased population towards people more comfortable in utilizing technology to communicate. Those who completed the survey may also not be representative of all people with a mental illness living in the US. There may be differences between those who decided to participate in the survey and those who chose not to. We need also to consider that as with any self-reported survey, there may be differences between how people answer the survey and how they feel. Finally, as the survey was conducted on-line with member and followers of ForLikeMinds, the survey most likely didn't capture the opinion of people without access to the internet or with little interest and/or knowledge of internet navigation.

\section{Conclusion}

The disruption caused by the COVID19 pandemic in mental health care may have not been completely solved by simply substituting tele-mental health care for in-person care. This survey offers an opportunity to reflect about the importance of building innovative strategies to create a working alliance with people who need care through tele-mental health. It is not mostly about the quantity of time providers spend with their clients on the phone or through video connections. It is about empowering and providing people the means to utilize technology as a tool ultimately to support them in their own recovery, including a focus on self-care and fostering social support. It is about the possibility of redefining ways in which tele-mental health is offered equitably and truly made accessible to everyone who needs it.

\section{References}

Chakrabarti, S. (2015). Usefulness of telepsychiatry: A critical evaluation of videoconferencing-based approaches. World Journal of Psychiatry, 5(3), 286-304. https://doi.org/10.5498/wjp.v5.i3.286.

Costa, M., Pavlo, A., Reis, G., Ponte, K., Davidson, L. (2020) COVID19 Concerns Among Persons with Mental Illness. Psychiatric Services. In the press. https://doi.org/https://doi.org/10.1176/ appi.ps.202000245.

Davidson, L. (2003). Living outside mental illness: Qualitative studies of recovery in schizophrenia. New York: New York, University Press.

Davidson, L., Wieland, M., Flanagan, E., \& Sells, D. (2008). Using qualitative methods in clinical research. Handbook of research methods in abnormal and clinical psychology (pp.253-270). Los Angeles: Sage Publications.

Dorsey, E. R., \& Topol, E. J. (2016). State of Telehealth. The New England Journal of Medicine, 375(2), 154-161. https://doi. org/10.1056/NEJMra1601705.

Greenhalgh, T., Wherton, J., Shaw, S., \& Morrison, C. (2020). Video consultations for covid-19. BMJ, 368, m998. https://doi. org/10.1136/bmj.m998.

Greenhalgh, T., Shaw, S., Wherton, J., Vijayaraghavan, S., Morris, J., Bhattacharya, S., et al. (2018). Real-World Implementation of video outpatient consultations at macro, meso, and micro levels: Mixed-method study. Journal of Medical Internet Research, 20(4), e150. https://doi.org/10.2196/jmir.9897.

Hilty, D. M., Ferrer, D. C., Parish, M. B., Johnston, B., Callahan, E. J., \& Yellowlees, P. M. (2013). The effectiveness of telemental health: a 2013 review. Telemedicine Journal and e-health: The Official Journal of the American Telemedicine Association, 19(6), 444-454. https://doi.org/10.1089/tmj.2013.0075.

Horowitz, L. M., Wilson, K. R., Turan, B., Zolotsev, P., Constantino, M. J., \& Henderson, L. (2006). How interpersonal motives clarify the meaning of interpersonal behavior: a revised circumplex model. Personality and social psychology review an official journal of the Society for Personality and Social Psychology, 
Inc, 10(1), 67-86. https://doi.org/https://doi.org/10.1207/s 1532 7957pspr1001_4.

Jenkins-Guarnieri, M. A., Pruitt, L. D., Luxton, D. D., \& Johnson, K. (2015). Patient perceptions of telemental health: Systematic review of direct comparisons to in-person psychotherapeutic treatments. Telemedicine Journal and e-health: The Official Journal of the American Telemedicine Association, 21(8), 652-660. https ://doi.org/10.1089/tmj.2014.0165.

Langarizadeh, M., Tabatabaei, M. S., Tavakol, K., Naghipour, M., Rostami, A., \& Moghbeli, F. (2017). Telemental health care, an effective alternative to conventional mental care: A Systematic review. Acta Informatica Medica: AIM : Journal of the Society for Medical Informatics of Bosnia \& Herzegovina: Casopis Drustva za Medicinsku Informatiku BiH, 25(4), 240-246. https://doi. org/10.5455/aim.2017.25.240-246.

Mehrotra, A., Huskamp, H. A., Souza, J., Uscher-Pines, L., Rose, S., Landon, B. E., et al. (2017). Rapid growth in mental health telemedicine use among rural medicare beneficiaries. Wide Variation Across States. Health Affairs (Project Hope), 36(5), 909-917. https://doi.org/10.1377/hlthaff.2016.1461.

Mahmoud, H., Whaibeh, E., \& Mitchell, B. (2020). Ensuring successful telepsychiatry program implementation: Critical components and considerations. Current Treatment Options in Psychiatry, 7(2), 186-197. https://doi.org/10.1007/s40501-020-00208-w.

Ponte K: Coronavirus: Mental Health Coping Strategies. https://www. nami.org/Blogs/NAMI-Blog/March-2020/Coronavirus-Menta 1-Health-Coping-Strategies Accessed 22 June 2020

Sevelius, J. M., Gutierrez-Mock, L., Zamudio-Haas, S., McCree, B., Ngo, A., Jackson, A., et al. (2020). Research with marginalized communities: Challenges to continuity during the COVID-19 Pandemic. AIDS and behavior, 24(7), 2009-2012. https://doi. org/10.1007/s10461-020-02920-3.

Shore, J. H. (2013). Telepsychiatry: videoconferencing in the delivery of psychiatric care. The American Journal of Psychiatry, 170(3), 256-262. https://doi.org/10.1176/appi.ajp.2012.12081064.

Shore, J. H., Yellowlees, P., Caudill, R., Johnston, B., Turvey, C., Mishkind, M., et al. (2018). Best practices in videoconferencing-based telemental health april 2018. Telemedicine Journal and e-health: The Official Journal of the American Telemedicine Association, 24(11), 827-832. https://doi.org/10.1089/tmj.2018.0237.

Siedner, M. J., Harling, G., Reynolds, Z., Gilbert, R. F., Venkataramani, A., \& Tsai, A. C. (2020). Social distancing to slow the US COVID19 epidemic: An interrupted time-series analysis. medRxiv.

Smith, A. C., Thomas, E., Snoswell, C. L., Haydon, H., Mehrotra, A., Clemensen, J., \& Caffery, L. J. (2020). Telehealth for global emergencies: Implications for coronavirus disease 2019 (COVID19). Journal of telemedicine and telecare, $1357633 X 20916567$.

Swank, J. M., \& Mullen, P. R. (2017). Evaluating evidence for conceptually related constructs using bivariate correlations. Measurement and Evaluation in Counseling and Development, 50(4), 270-274. https://doi.org/10.1080/07481756.2017.1339562.

Torous, J., Jän Myrick, K., Rauseo-Ricupero, N., \& Firth, J. (2020). Digital mental health and COVID-19: Using technology today to accelerate the curve on access and quality tomorrow. JMIR Mental Health, 7(3), e18848. https://doi.org/10.2196/18848.

Van Manen, M. (2016). Researching lived experience: Human science for an action sensitive pedagogy. Albany, N.Y.: State University of New York Press.

Wright, J. H., \& Caudill, R. (2020). Remote treatment delivery in response to the COVID-19 pandemic. Psychotherapy and Psychosomatics, 89(3), 130-132. https://doi.org/10.1159/000507376.

Yellowlees, P., Shore, J., Roberts, L., \& American Telemedicine Association. (2010). Practice guidelines for videoconferencing-based telemental health - October 2009. Telemedicine Journal and e-health: the official journal of the American Telemedicine Association, 16(10), 1074-1089. https://doi.org/10.1089/tmj.2010.0148.

Publisher's Note Springer Nature remains neutral with regard to jurisdictional claims in published maps and institutional affiliations. 